\title{
Integrated Approach to Power Distribution Loss Based on Improved Genetic Algorithm
}

\author{
Chen $\mathrm{Li}^{1}{ }^{1, *}$, Yunsi Huang ${ }^{2}$, Anjia Mao ${ }^{1}$, Yu Zhang ${ }^{3}$ and Peng $\mathrm{Wu}^{3}$ \\ ${ }^{1}$ North China Electric Power University, Beijing, China \\ ${ }^{2}$ State Grid Economic and Technical Research Institute, Beijing, China \\ ${ }^{3}$ State Grid Energy Research Institute Co., Ltd., Beijing, China \\ ${ }^{*}$ Corresponding author
}

\begin{abstract}
Profit space of power grid enterprises is being compressed under the background of continuous improvement of power system reform in China. At the same time, due to environmental and climate issues, the state strongly advocates energy conservation and emission reduction, all of which makes the grid enterprises pay more attention to the loss of power grid than before. In this paper, in the case of no additional investment, we use the existing distribution network equipment, a comprehensive quantitative distribution network, network reconfiguration and reactive power compensation for transformer economic operation on the influence of network loss. In this paper, a mathematical model of power loss reduction is established based on the minimum power dissipation. To improve the mutation rate and cross rate to improve the genetic algorithm, using the algorithm to solve the model, the algorithm's search performance has been improved considerably.
\end{abstract}

Keywords-distribution network reconstruction; transformer economy operation; improved genetic algorithm

\section{INTRODUCTION}

For a long time, the focus of the power grid construction in China are mainly concentrated in high technical content, play a key role for the safe and stable operation of power supply capacity and the transmission of the Internet, insufficient attention and relatively simple construction, small power supply scope of $10 \mathrm{kV}$ and below the terminal distribution network. In the reality, the distribution network is complicated, and the main network loss in the distribution network is concentrated in the distribution network, which influences the economy of power system operation.

Different from the traditional vertical integration business model, in the new market economy environment, the power industry is more competitive, and the profit space of power grid enterprises will be compressed with the accounting of the transmission and distribution price. Therefore, the refined management mode of open source saving, improving investment and operating efficiency is accepted by more and more power grid enterprises. Network loss as an important index of power transmission and distribution economy level, reduces network loss to become an important "throttling" measure of power grid enterprises in the new environment. In addition, the state has started to advocate energy conservation and emission reduction and improving energy efficiency is an effective strategy for power grid enterprises to respond to emission reduction policies. Therefore, the loss of transmission and distribution lines is an important link in the planning and operation optimization of power grid.

The purpose of this paper is to optimize the operation to achieve the purpose of loss reduction without additional investment. Literature 1 proposed a new evolutionary programming mutation operator, which improved the calculation speed of the whole distribution network reconstruction algorithm. In the literature 2, a reactive power optimization mathematical model with minimum annual cost as the objective function was established, and a subgroup particle swarm algorithm with fusion fission and mutation operation was proposed to solve the model. Literature 3 of the automatic control system with load capacity and analyzed the design and implementation methods of key control strategies such as economic operation strategy and safe operation strategy. The main measures to reduce the loss of the current distribution network technology are to use network reconstruction and reactive power compensation to optimize the distribution network operation, and to reduce the loss of transformer through the economic operation of the transformer. In recent years, there has been some research on the effects of these two kinds of damage reduction measures on economic model. For example, literature 4 adopts the alternate iteration method of reactive power compensation after network reconstruction. In the literature 5, an alternate iteration algorithm based on the electrical dissection information is proposed, which is fast in computation and good in convergence. However, these algorithms are based on a partial optimal solution, which is a partial optimal solution, which makes it easy to get into a local optimal solution, and it's difficult to get the optimal solution. In many algorithms, genetic algorithm because the code is simple, does not require the objective function has good mathematical properties, and based on elite reserved strategy, is a kind of global convergence of the algorithm in theory, and therefore different aspects in the power system has been widely used. More details can be found in the literature 6 .

In this paper, a mathematical model is established to comprehensively consider the reconstruction of distribution network, reactive power compensation and transformer economic operation, to optimize the value of crossover rate and mutation rate in traditional genetic algorithm, to improve the search performance of the traditional genetic algorithm, and to solve the model established by the improved genetic algorithm. 
Finally, the simulation experiment of IEEE-33 system is carried out to verify the effectiveness of this model in reducing the loss of power distribution network and the advantages of single or two reduction measures.

\section{MATHEMATICAL MOdEL OF THE INTEGRATED REDUCTION METHOD}

The transformer economic operation considered in this paper is to make the transformer operate near the active economic load coefficient. The objective function of the circuit and transformer active network loss is shown in the following formula:

$$
\min f=\sum k_{i} \Delta P_{l i}+\Delta P_{T}==\sum_{i=1}^{N} k_{i} R_{i} \frac{P_{l i}^{2}+Q_{l i}^{2}}{U_{i}^{2}}+P_{0}+\beta^{2} P_{k}
$$

Among them, $\mathrm{N}$ is the number of branches; $\Delta P_{l i}$ is the active loss of line $\mathrm{i}$; $k_{i}$ is the state of switch $\mathrm{i}$ ( 0 is open, 1 is closed); $R_{i}$ is the resistance of branch $\mathrm{i} ; P_{l i}$ is the active trend of line $\mathrm{i} ; Q_{l i}$ is the reactive power flow of branch $\mathrm{i}$ after adding reactive power compensation; $U_{i}$ is the voltage value of line i; $\Delta P_{T}$ is the active loss of transformer; $P_{0}$ and $P_{k}$ are the no-load losses and short-circuit losses of transformers; $\beta$ for transformer load rate, as the reactive power compensation capacity and the network loss varies, mainly by the influence of the reactive power compensation capacity.

Equation constraint equation is the power balance equation, namely:

$$
\left\{\begin{array}{l}
P_{i}=U_{i} \sum_{j=1}^{n} U_{j}\left(G_{i j} \cos \theta_{i j}+B_{i j} \sin \theta_{i j}\right) \\
Q_{i}+Q_{c i}=U_{i} \sum_{j=1}^{n} U_{j}\left(G_{i j} \sin \theta_{i j}-B_{i j} \cos \theta_{i j}\right)
\end{array}\right.
$$

Among them, $P_{i}$ represents the injected active power of node $\mathrm{i} ; Q_{i}$ represents the injected reactive power of node $\mathrm{i}$; $Q_{c i}$ represents the reactive power compensation capacity of node $\mathrm{i} ; U_{i}, U_{j}$ represents voltage amplitude of node $\mathrm{i}$ and $\mathrm{j}$; $G_{i j}$ and $B_{i j}$ represent the conductance and susceptance between node i and $\mathrm{j}$; $\theta_{i j}$ represents the phase angle difference between node $\mathrm{i}$ and $\mathrm{j}$.

Inequality constraints are as follows:

$$
\left\{\begin{array}{l}
U_{i \min } \leq U_{i} \leq U_{i \max } \\
Q_{c \min } \leq Q_{c i} \leq Q_{c \max } \\
S_{j} \leq S_{j \max }
\end{array} \quad\left(\begin{array}{c}
i=1,2, \mathrm{~K}, n \\
j=1,2, \mathrm{~K}, N
\end{array}\right)\right.
$$

In the above equation, $U_{i \min }$ and $U_{i \min }$ represent the lower limit and upper limit of the allowable voltage of node $i$; $Q_{c \min }$ and $Q_{c \max }$ represent the lower limit and upper limit value of each compensation point. $S_{j}$ and $S_{j \max }$ represent the power and maximum allowable power of the feeder; $\mathrm{N}$ and $\mathrm{n}$ represent the number of branches and nodes of the system.

The mathematical model established in this paper is a combination of nonlinear optimization and integer optimization. It is difficult to solve the problem with traditional mathematics, and the genetic algorithm is less affected by the target function, so the algorithm is used to solve the problem.

\section{IMPROVED GENETIC ALGORITHM FOR LOSS REDUCTION}

The traditional genetic algorithm has the disadvantage of slow convergence speed and difficult to obtain the global optimal solution. This paper mainly improves the algorithm by optimizing the mutation rate and the crossover rate.

\section{A. Chromosome Coding}

This article uses binary encoding. The " 0 " or " 1 " represents the state of the line switch in the network, and the length of the chromosome is the total number of switches. In reactive power optimization, chromosome available [G1, G2,..., Gi,..., Gm], said $m$ represents the need for reactive power compensation of the total number of nodes in network, Gi on behalf of the length of binary string of ki represents the node compensation after converted to a decimal number of capacitor Banks, binary code simply cover it maximum compensation capacity. To connect the remodeling to the unreactive chromosomes, it forms the chromosomes that are required to reduce the loss.

\section{B. Selection of Crossover and Mutation Rates}

Due to the addition of the gene segment of reactive power compensation after the reconstruction of the distribution network, the chromosome length of the whole participating genetic algorithm is increased, and the search space of the algorithm is expanded, which affects the search efficiency of the algorithm to a certain extent. In order to improve the search performance of the algorithm, the crossover and mutation rates can be optimized appropriately.

In the early iteration of genetic algorithm, the individual randomness is large and the quality is poor, so the search speed of the algorithm is guaranteed by using a large cross rate. However, in the later period of the iteration, when the individual tends to the optimal solution, the high cross rate can destroy the good genes. Of course, cross rates cannot be too small to affect the genetic search speed. Therefore, the size of the cross-rate $P_{c}$ should be modified according to the good 
degree of individual population in the iteration process. The formula adopted in this paper is as follows:

$$
P_{c}=\left\{\begin{array}{lc}
P_{c s} & \bar{F}^{(\mathrm{n})}>\varepsilon, P_{c}>P_{c b} \\
P_{c s}-\frac{\bar{F}^{(\mathrm{m})}-\bar{F}^{(\mathrm{n})}}{\bar{F}^{(0)}} \alpha n & \bar{F}^{(\mathrm{n})} \leq \varepsilon, P_{c}>P_{c b} \\
P_{c b} & P_{c} \leq P_{c b}
\end{array}\right.
$$

Among them, $\mathrm{N}$ is the algebra of this iteration; $\mathrm{M}$ is the iterative algebra for initial conditions of $\bar{F}^{(\mathrm{n})} \leq \varepsilon ; P_{c s}$ represents the maximum cross rate, namely the initial cross rate; $P_{c b}$ represents the minimum cross rate; $\alpha$ is to reduce the step parameters for the crossover rate; $\varepsilon$ is the limit of the fitness difference needed to reduce the cross rate; $\bar{F}^{(0)}, \bar{F}^{(\text {n) }}$ and $\bar{F}^{(m)}$ respectively represent the fitness average of initial population, the $\mathrm{n}$ th iteration population and the $\mathrm{m}$ th iteration population. Because the value range of cross rate is generally $0.4 \sim 0.9, P_{c s} 、 P_{c b} \in[0.4,0.9]$.

The curve in Figure I simulates the change trend of the discrete cross rate. The variable meaning in the figure is the same as equation (4):

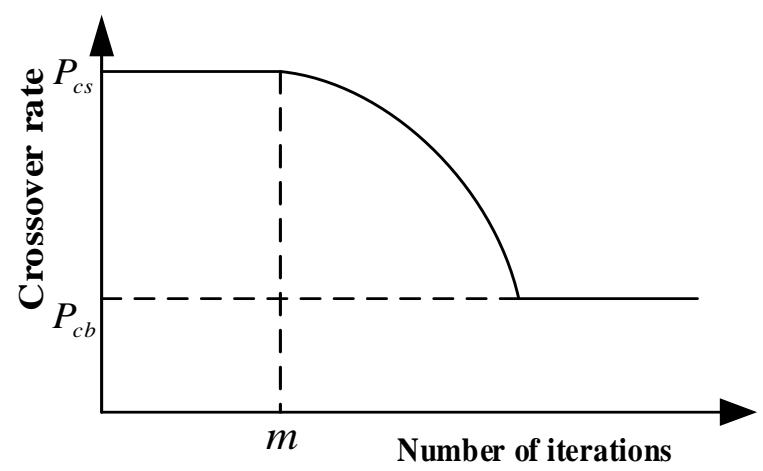

FIGURE I. SIMULATION CURVE OF CROSS-RATE

The optimal range of the variation rate is $0.001 \sim 0.1$. In the later stages of the genetic algorithm, the populations vulnerable to the same chromosome, cross may be the same between two chromosomes, the cross has lost its meaning, and once appear, the optimal chromosome successive generations of the same situation, make the algorithm falls into local optimum easily, so you need to further improve the mutation rate. The variation rate set in this paper is as follows:

$$
P_{m}=\left\{\begin{array}{lc}
P_{m s} & \bar{F}^{(\mathrm{n}+1)}-\bar{F}^{(\mathrm{n})}<\delta \\
P_{m b}+\gamma n & \text { others }
\end{array}\right.
$$

Among them, $\gamma$ is the step length with increasing mutation rate; $\delta$ is the limit of the difference between the average fitness of the neighboring two generations; $P_{m s}$ and $P_{m b}$ represent the maximum mutation rate and the minimum mutation rate respectively.

The curve in Figure II simulates the variation trend of the discrete variation rate, and the meaning of the variable in the figure is the same as that of equation (5):

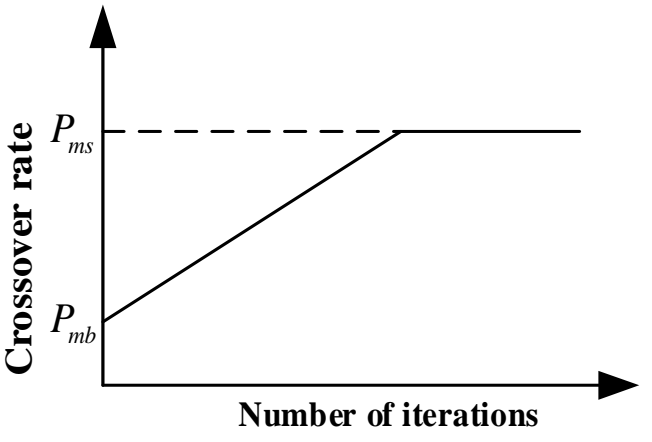

FIGURE II. SIMULATION CURVE OF MUTATION RATE

\section{Main Steps of the Algorithm}

1) Set initial parameters of chromosome encoding length $L$, population size $N_{0}$, population maximum evolutionary algebra $N_{G}$, etc.

2) Generate initial population $X_{0}$ of $N_{0}$, repair the unfeasible solution, and set genetic algebra $T=1$.

3) Network power flow calculation, and the fitness of chromosome.

4) Genetic manipulation and sequencing.

5) Determine whether the convergence condition is met. If it is, then the first individual of the output population, if not, then $T=T+1$, and then go to step 3 ).

\section{NUMERICAL EXAMPLE}

Based on the Matlab platform, the simulation calculation of the IEEE-33 node system is carried out, as shown in Figure III. The positive body number represents the node number and the italic number represents the branch number. The system consists of 37 branches, of which 5 are the contact switch branches, the reference voltage is 12.66 kilovolts, the base power is 10 megawatts, and the total network initial state load is $4084.26+\mathrm{j} 2547.32$ kilovolt amperes. Assuming that the double winding transformer is connected to the node 1 , its model is sfz7-6300/110, the rated capacity is 6300 kilovolt amperes, and the parameters are:

$$
\begin{aligned}
& P_{k}=41 \text { kilowatts, } P_{0}=12.5 \text { kilowatts } \\
& I_{0} \%=1.4, U_{k} \%=10.5
\end{aligned}
$$




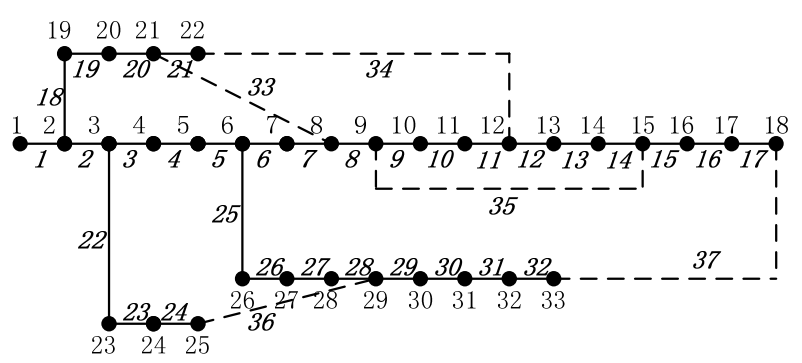

FIGURE III. IEEE-33 NODES DISTRIBUTION NETWORK

The method of this paper is compared with three kinds of loss reduction schemes:

Plan $1 . \quad$ Optimization of reactive power compensation alone.

Plan 2. Reconfiguration of distribution network separately.

Plan 3. Reconfiguration of distribution network and reactive power compensation, but not the economic operation of transformer.

We use the nodes 30, 8 and 14 as reactive compensation points, and each compensation capacity is shown in the table, and the unit is kilovar. The advantages of this scheme are demonstrated by comparing the effects of different schemes. Compared with the plan 1 , it can be seen that the scheme compensation capacity of this paper is low and the loss reduction rate is high. This is because this paper has optimized the active and reactive flow of the system after the reconfiguration of the distribution network. On the one hand, the compensation capacity has been reduced, and on the other hand, the system network loss is smaller. The effect of reactive power compensation on reducing network loss can be seen by comparing with plan 2. The comparison with plan 3 highlights the importance of transformer economic operation. The detailed comparison is shown in Table I.

TABLE I. COMPARISON OF POWER GRID LOSS REDUCTION

\begin{tabular}{|c|c|c|c|c|}
\hline Type & Plan1 & Plan2 & Plan3 & $\begin{array}{c}\text { Our } \\
\text { algorithm }\end{array}$ \\
\hline Node 30 (kilovar) & 930 & - & 920 & 1040 \\
\hline Node 8 (kilovar) & 300 & - & 280 & 280 \\
\hline Node 14 (kilovar) & 390 & - & 320 & 240 \\
\hline $\begin{array}{c}\text { Loss before optimization } \\
\text { /p.u. }\end{array}$ & 0.0203 & 0.0207 & 0.0239 & 0.0239 \\
\hline $\begin{array}{c}\text { Loss after optimization } \\
\text { /p.u. }\end{array}$ & 0.0147 & 0.0142 & 0.0131 & 0.0123 \\
\hline Loss reduction rate & 0.2759 & 0.3149 & 0.4519 & 0.4854 \\
\hline
\end{tabular}

\section{CONCLUSION}

In this paper, the influence of multiple factors on the loss of power distribution network is taken into account, and the improved genetic algorithm is used to solve the problem. The model is applied to the simulation experiment in the IEEE-33 node system, and the following conclusions can be drawn from the experimental results:

(1) In this paper, the optimization and improvement of the mesh reduction model is carried out, and the reduction results are more comprehensive after the comprehensive distribution network reconstruction, reactive power compensation and transformer integrated operation.

(2) In this paper, we propose a genetic algorithm that considers the reactive power compensation into binary chromosome coding and optimize and correct the crossover and mutation rates used in the algorithm, making optimization search more efficient.

(3) The method proposed in this paper can effectively reduce the loss of power distribution network, and the loss effect is more obvious than that of single or two reduction measures.

\section{ACKNOWLEDGMENT}

This work was supported by 2017 Science and Technology Project of State Grid Corporation of China (Research on electric energy substitution planning models, policy simulations and their applications, No. 52130N17000R)

\section{REFERENCES}

[1] ZHANG Bingda, LIU, "A Distribution Network Reconfiguration Algorithm Based on Evolutionary Programming Mutation Operator”. vol. 36, Power System Technology, 2012, pp. 202 206.

[2] ZHANG Tingchang, GENG Guangfei, "Reactive Power Optimization for Medium Voltage Distribution Network Based on Improved Particle Swarm Optimization”. vol. 36, Power System Technology, 2012, pp 158-162.

[3] FAN Wenbo, HAN Shaigen, "A Control Strategy for Secure and Economic Operation of On-load Capacity Regulating Transformer,” Automation of Electric Power Systems, vol. 35, pp. 98-102, 2011.

[4] Deng Youman, Zhang Boming, Wang Hongpu. “A comprehensive optimization algorithm for distribution network reconfiguration and capacitor switching,” Automation of Electric Power Systems, vol. 20, pp. 5-9, 1996.

[5] Hao Wenbo. "Study on the optimization method of distribution network restricted by electrical rules,” Harbin Institute of Technology, 2007.

[6] CHEN Gonggui, LI Zhihuan, SUN Yong-fa, ZHONG Jianwei. "LRSPSO Algorithm for Optimal Reactive Power Flow," Automation of Electric Power Systems, vol. 20, pp. 92-97, 2008. 